\title{
Bullous Skin Reaction after Blood Transfusion: A Case Presentation.
}

\author{
Ahmet Yüksek ${ }^{1^{*}}$ \\ ${ }^{1}$ Department of Anesthesiology and Reanimation, Bozok University, Yozgat, Turkey \\ *Corresponding Author: Ahmet Yüksek, Department of Anesthesiology and Reanimation, Bozok University, Yozgat, Turkey \\ Received date: 25 February 2021; Accepted date: 06 March 2021; Published date: 01 August 2021 \\ Citation: Ahmet Yüksek (2021) Bullous Skin Reaction after Blood Transfusion: A Case Presentation. J Med Case Rep Case Series 2(8): \\ https://doi.org/10.38207/JMCRCS/2021/020803
}

Copyright: (C) 2021 Dr. Ahmet Yüksek. This is an open-access article distributed under the terms of the Creative Commons Attribution License, which permits unrestricted use, distribution, and reproduction in any medium, provided the original author and source are credited.

\begin{abstract}
Intensive care is one of the most common sites where blood transfusions are performed. Although anaphylactic reactions due to blood transfusions are not common, milder reactions and skin reactions are common. However, bullous reaction after blood transfusion is very rare. In this case, bullous skin reaction was observed after a unit of red cell suspension has been transfused to an autoantibody positive patient. Fortunately, the patient's response did not progress, and no vital problems occurred in the patient's follow-up. Therefore, we wanted to share this rare blood transfusion reaction to contribute to the literature
\end{abstract}

Keywords: Blood transfusion; Bullous skin reaction; Skin reactions.

\section{Introduction}

Blood transfusions are used in surgical and trauma patients for rapid correction of severe anemia caused by bleeding or in the treatment of internal bleeding or coagulation disorders. Blood products can be used as whole blood or separated into components [1]. Blood transfusions organization in Turkey is carried out by the Turkish Red Crescent which is established in 1957 [2]. The collected blood is divided into subunits and is distributed to hospitals and centers after being examined for infection carriers [3]. Since the detection of ABO isoagglutinin by Landsteiner in 1901, the safety and chances of success in blood transfusions have increased dramatically [4] Screening and comparison tests for safe blood transfusion are routinely performed. The adverse effects of unnecessary blood transfusion on the patient and the cost are now well known. Although it is accepted as 7-8 $\mathrm{g} / \mathrm{dl}$ in restrictive strategy and 9-10 $\mathrm{g} / \mathrm{dl}$ according to liberal strategy, the threshold value for blood transfusion varies according to the patient's condition [5]. However, the common point of both strategies is that the risks for patients increase when blood transfusion is unnecessary.

\section{Case History}

An 83-year-old female patient was brought to the emergency room within 20 minutes after cardiac arrest. The patient was returned to sinus rhythm with the intervention in the emergency department, but her Glasgow coma score did not increase. The patient was followed by the diagnosis of ischemic encephalopathy. She had no known disease other than COPD and had not undergone a blood transfusion before. The patient was admitted to the anesthesia intensive care unit for follow-up and treatment. Hemoglobin value of 9.5 in the intensive care was decreased to $8 \mathrm{~g} / \mathrm{lt}$ on the $27 \mathrm{th}$ day of follow-up. The hematocrit value of the patient was 25 . The vital values of the patient
The riskiest conditions related to blood transfusion include transfusion-transmitted bacterial infection, transfusion-related acute lung injury (TRALI), transfusion-associated circulatory overload (TACO) and anaphylactic reactions [6]. Fortunately, these major events also decreased with transfusion safety and reduced off-label use. However, allergic reactions such as transfusion-related rash and purpura are common. In a study of 1613 patients, anaphylactoid reactions were observed in $7.7 \%$ and allergic reactions were observed in $17 \%$ [7]. Among the transfusion-induced skin reactions, purpura is the most common. In this case, we report a case of a bullous skin reaction after blood transfusion to an autoantibody-positive patient. According to the literature, bullous reactions after blood transfusion are not common skin findings. Even, we have not seen any cases of bullous reaction due to blood transfusion in our literature search. We aimed to share our case which we thought was interesting in terms of being a first.

were blood pressure 90/55, heart rate 122, saturation $88 \%$, under mechanical ventilation respiratory rate 10 / minute, tidal volume 540 and $\mathrm{fio}_{2}$ was $50 \%$. Upon the patient was hypotensive and tachycardic one unit of erythrocyte suspension transfusion was planned. The patient had no fever, had previously undergone analgesics, and had not received any other medication within the last hour. Blood group and blood crossmatch at the blood bank was requested with the blood sample of the patient. The blood group was $0 \mathrm{Rh}+$ and positive for the auto-antibody test. (Figure 1). 


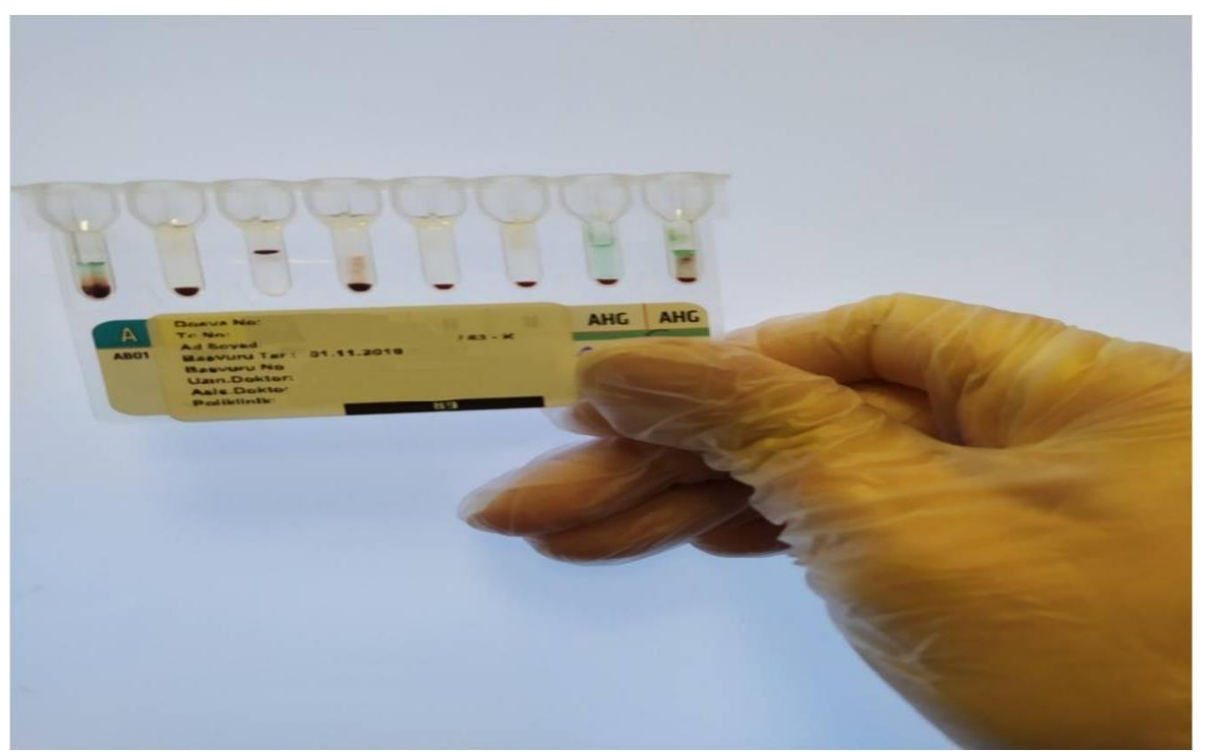

Figure 1: Blood results with the crossmatch test. The reaction in the rightmost tube with antibodies in the patient's blood is observed.

The transfusion team was consulted on the fact that the autoantibody that was positive in the patient's blood tests. The transfusion team indicated that autoantibody subtyping was not performed and was not required in routine procedures. Whereat, one unit of erythrocyte suspension was started at $100 \mathrm{ml} / \mathrm{hr}$ via central venous catheter from an internal jugular vein. At the 20th minute of the transfusion, many bullae were observed in the chest area (Figure 2). These bullae did not exceed $1 \mathrm{~cm}$ and spread on both shoulders and from the chest to the upper abdomen. Transfusion was stopped and methylprednisolone $1 \mathrm{mg} / \mathrm{kg}$ and pheniramine $45.5 \mathrm{mg}$ were administered to the patient. The allergic reaction that rapidly regressed after the first treatment and reported to the transfusion committee. The patient's blood transfusion was then continued with a slower rate of $40 \mathrm{ml} / \mathrm{hr}$, and transfusion was completed.

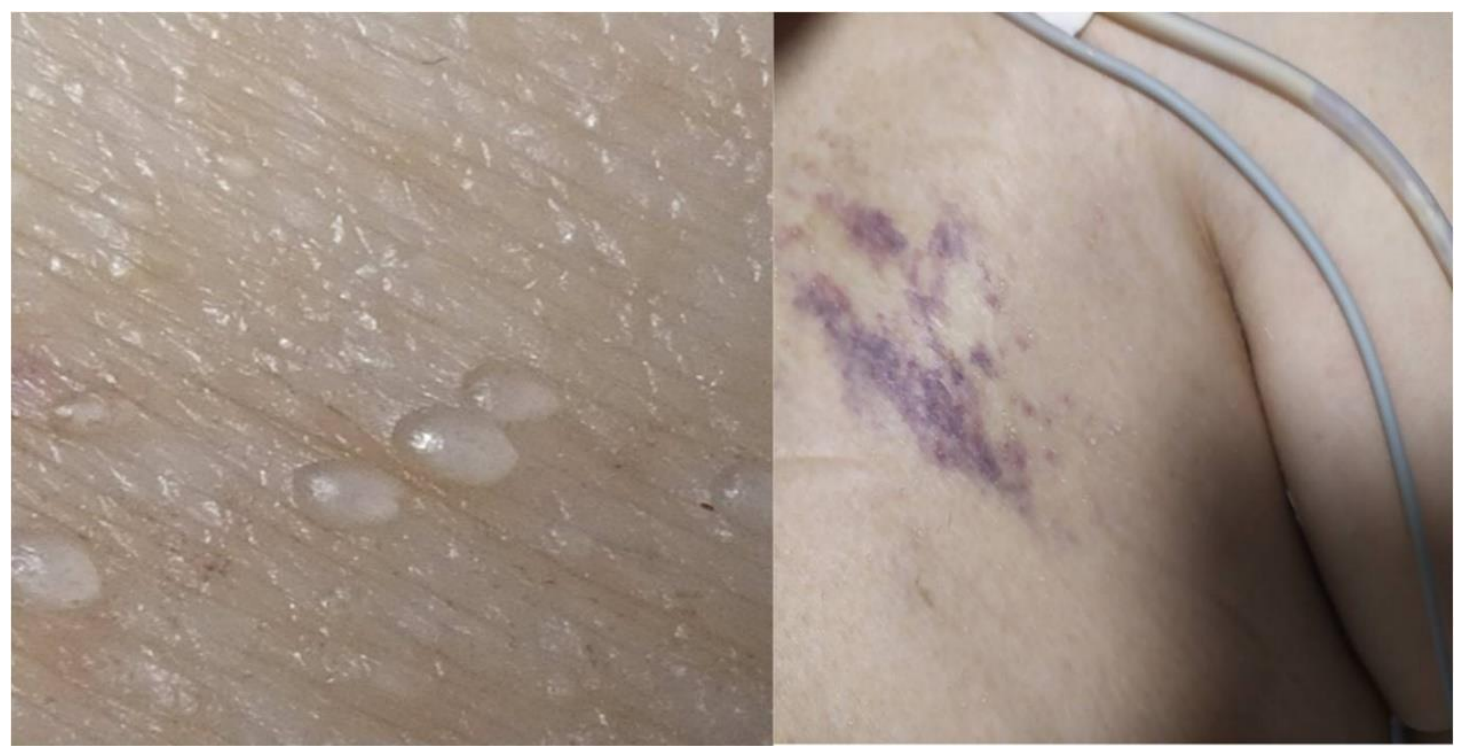

Figure 2: Bullae in the patient's chest. The image on the right is the patient's upper left chest area, the image on the left is the zoomed image of the same region.

\section{Results and Discussion}

In this case, an uncommon transfusion reaction was shared. Although allergic reactions are common after transfusion, the bullous reaction was not observed in our literature search. In this respect, it is important to be the first case we have reported. In addition, the decision stage of the blood transfusion, the tests performed, the site and the speed of application are the cases that should be discussed with their right and wrong sides.

In this case, the patient was given a blood transfusion at a hemoglobin value of $8.0 \mathrm{~g} / \mathrm{dl}$. This value is between the transfusion thresholds known as liberal or restrictive strategy. However, advanced age, hypotensive and tachycardic course was effective in the decision of transfusion. In this case, it is based on the idea that transfusion thresholds should be individually determined for each patient and have no sharp limits.
Most medical centers perform pre-transfusion tests for safe transfusions, such as $\mathrm{ABO}, \mathrm{RhD}$ blood typing, and crossmatch. Nevertheless, the occurrence of transfusion reactions due to unexpected antibodies other than ABO blood type antibody and anti$\mathrm{D}$ antibody may not be predicted by these tests alone, and therefore screening and detection tests of rare antibodies may be required but are not performed in routine procedures. The autoantibody test was reported as positive before transfusion in patient blood. Autoantibodies are frequently observed in surgical patients and autoimmune diseases, especially after frequent blood transfusions. Typing of these antibodies is not routinely performed, but in terms of cost is a matter to be discussed. Also, it was on the 27th day of our patient's admission and blood transfusion had not been done before. She also had no known autoimmune disease. In principle, blood 
transfusions through peripheral vessels are recommended for observing allergic reactions. Peripheral edema and recurrent vascular access in patients hospitalized in the intensive care unit for a long period of time may lead to unusable states of the peripheral vessels. The use of a central catheter for blood transfusion is not uncommon in these patients and in patients who require rapid blood transfusion perioperatively. However, allergic, and anaphylactic reactions that occur at this time may be more severe. In our patient, blood transfusion was planned to end in 2 hours and then the transfusion rate was further reduced. In autoantibody-positive patients, careful and slower transfusion continuation is the practice in our clinic. Fortunately, the patient's prognosis was good, there were no other signs of transfusion reaction. However, it is a fact that a similar

\section{Conflict of interest: None}

\section{References}

1. Connell NT (2016) 'Transfusion Medicine', Prim Care. 43(4): 657-659.

2. Kan Transfüzyonunun and others, 'Kan-TransfuzyonununTarihcesi-Tanju-Atamer', 1957, 148-54.

3. Deveci O, Tekin A, Günbay SS, Kılıç D, Kaygusuz S, et al. (2011) 'Evaluation of HBsAg, Anti-HCV, Anti-HIV and VDRL Test Results in Blood Donors', Journal of Clinical and Experimental Investigations, 2(4): 416-419.

4. Christopher Lattimer and Chris Perera, 'Blood Transfusion', in Key Topics in General Surgery, 2002

5. Carson JL, Stanworth SJ, Roubinian N, Fergusson DA, Triulzi D, et al. (2016) 'Transfusion Thresholds and Other Strategies for situation cannot be completely prevented in the future and such reactions cannot be predictable. What makes our case interesting is that the patient reacts to the transfusion with bullae. Allergic reactions are observed in $1 \%$ of all transfusions [8]. The most common transfusion-related skin reactions are purpura, urticaria and anaphylactoid reactions, and the frequency of the transfusion-related purpura is estimated at 1 / 200,000. World Health Organisation classified transfusion-related skin findings and the bullous reaction is not in this classification [8]. In this case, we shared a patient who had a bullous skin reaction in one patient after erythrocyte transfusion. We believe that this situation, which is not common in the literature and clinical practice, therefore our case is different for those interested in the subject and is probably the first case.

\section{Acknowledgment: None}

\section{Source(s) of support: None}

Guiding Allogeneic Red Blood Cell Transfusion', Cochrane Database Syst Rev. 10(10): CD002042.

6. Therese A. Callaghan, 'Transfusion Reactions', in Haematology in Critical Care: A Practical Handbook. 2014.

7. Domen RE, Hoeltge GA (2003) Allergic Transfusion Reactions: An Evaluation of 273 Consecutive Reactions, Arch Pathol Lab Med. 127(3): 316-20.

8. Blood Transfusion Safety Team and World Health Organisation. The Clinical Use of Blood: Handbook. Who, Geneva, 2001; pp62-79. 